\title{
An Achievable Rate-Distortion Region for the Multiple Descriptions Problem
}

\author{
Farhad Shirani \\ EECS Department \\ University of Michigan \\ Ann Arbor,USA \\ Email: fshirani@umich.edu
}

\author{
S. Sandeep Pradhan \\ EECS Department \\ University of Michigan \\ Ann Arbor,USA \\ Email:pradhanv@umich.edu
}

\begin{abstract}
A multiple-descriptions (MD) coding strategy is proposed and an inner bound to the achievable rate-distortion region is derived for discrete memoryless sources. The scheme utilizes linear codes. It is shown in two different MD set-ups that the linear coding scheme achieves a larger rate-distortion region than previously known random coding strategies. Furthermore, it is shown via an example that the best known random coding scheme for the set-up can be improved by including additional randomly generated codebooks.
\end{abstract}

\section{INTRODUCTION}

The multiple-descriptions (MD) source coding set-up describes a communication system consisting of a centralized encoder and several decoders. The encoder transmits data through a number of noiseless links. Each decoder is connected to the encoder via a subset of these links. The goal is for the encoder to compress an information source and transmit it to the decoders such that the source reconstruction at each decoder meets a specific fidelity criterion. There has been an extensive amount of effort to determine the optimal ratedistortion (RD) region for the general MD set-up, however, even in the case of two-descriptions (see figure 1) the optimal region is not known. The best known achievable RD region for the two-descriptions set-up is due to Zhang and Berger [1]. In [1], the encoder utilizes a base layer which is decoded by all receivers and a refinement layer which is decoded by individual receivers. The VKG scheme proposed in [2] generalizes the base layer idea in [1] to cases with more than two-descriptions. The combinatorial-message-sharing (CMS) strategy [3] expands the method in [2] by considering a combinatorial number of base layer codebooks which are decoded in subsets of receivers. In [4] and [5], random binning schemes were introduced which result in gains over previous known coding strategies. The methods in [4] and [5] are only applicable to symmetric rate-distortion settings. Finally, in [6] the ideas in [3] and [4] were combined to form CMS with binning. It was shown that CMS with binning gives gains over previous coding schemes and strictly contains them. All of these schemes use random codes to construct codebooks; in this paper we propose using linear codes instead.

Structured codes in communication problems have traditionally been of interest due to their practicality in compar-

This work was supported by NSF grant CCF-1111061.

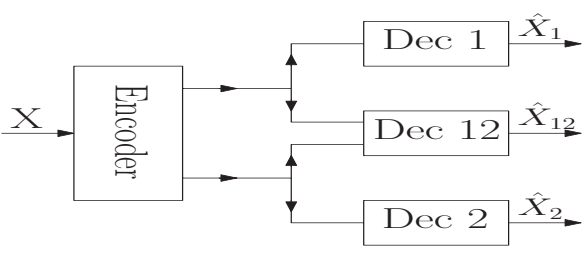

Fig. 1. The two-descriptions problem

ison with randomly generated codes. Korner and Marton [7] observed that in a specific distributed source coding set-up, application of structured codes may also yield gains in terms of achievable rate-distortions. The phenomenon was observed in other multi-terminal problems as well. It was shown in the three user interference channel [8], the three user broadcast channel [9], variations of the MAC channel [12], [13] and the three user distributed source coding problem [14], that employing linear codes results in gains. Intuitively, the main idea behind all of these linear coding schemes is that because of their structure, linear codes can compress and transmit sums of binary random variables more efficiently than random codes. Based on these observations it is expected that utilizing linear codes is also advantageous in the MD problems when more than 2 descriptions are transmitted. This turns out to be indeed the case as we illustrate in the following sections.

The rest of the paper is organized as follows: Section II is allocated to explaining the CMS with binning scheme. In section III, we prove that linear codes give gains over the CMS with binning strategy in two different examples. Section IV contains a proof that the CMS with binning scheme can be improved using random codes. In section $\mathrm{V}$, we provide an achievable RD region for the MD problem. Section VI concludes the paper.

\section{CMS WITH BINNING}

This section includes a brief summary of the CMS with binning scheme introduced in [6]. Let $A \subset[1: n]$ be a subset of indices, denote a set of random variables (RV) $\left(X_{i}\right)_{i \in A}$ as $X_{A}$. Define $P_{X_{A}}$ as the joint distribution on $X_{A}$.

Consider an L-descriptions problem. Let $X$ be a DMS with distribution $P_{X}$. For an $\underline{s} \in[1: L]$, let $d_{\underline{s}}$ be the distortion function at decoder $\underline{s}$. Let $\mathcal{S}=\{(A, j) \mid A \subset[1: L], j \in[1$ : 
$|A|]\}$ and $\mathcal{T}=\{(k, n) \mid k \in[1: L-1], n \in[1: L]\} . V_{\mathcal{S}}$ is the set of base layer RV's and $U_{\mathcal{T}}$ is the set of refinement layer RV's. Fix the joint probability distribution $P_{V_{\mathcal{S}}, U_{\mathcal{T}} \mid X}$.

Base Layer Construction: For each subset $A$ of $[1: L]$, construct $|A|$ codebooks $C_{A, i}, i \in[1:|A|]$. Each codebook is generated based on the marginal probability distribution $P_{V_{A, i}}$, independent of other codebooks. The codebook has rate $r_{A, i}$. This codebook is to be decoded if a decoder receives at least $i$ descriptions from the set $A$. For each description in $A$, the encoder bins the codebook at a different rate. Binning is performed for each description independent of other descriptions. The binning rate of codebook $C_{A, i}$ for description $j$ is $\rho_{A, i, j}$. Thus, bin size equals $2^{n\left(r_{A, i}-\rho_{A, i, j}\right)}$. On description $j \in A$, the encoder sends the bin number of the codeword from $C_{A, i}$, which requires rate $\rho_{A, i, j}$.

Refinement Layer Construction: For description $j$, construct $L-1$ refinement layer codebooks, $C_{i, j}, i \in[1: L-1]$. Each codebook is generated based on $P_{U_{i, j}}$ and has rate $r_{i, j}$. The codebook is decoded if the decoder receives description $j$ along with at least $i-1$ other descriptions (i.e. the codebook is an SCEC sent by encoder $j$ ). The codebook is binned at rate $\rho_{i, j}$.

Covering Bounds: Since the codebooks are generated independently, typicality requires mutual covering bounds for all subsets of random variables. Hence for all $\mathcal{A} \subset \mathcal{S}$ and $K \subset \mathcal{T}$ we must have:

$H\left(V_{\mathcal{A}}, U_{K} \mid X\right) \geq \sum_{(A, j) \in \mathcal{A}}\left(H\left(V_{A, j}\right)-r_{A, j}\right)+\sum_{(k, n) \in K}\left(H\left(U_{k, n}\right)-r_{k, n}\right)$

Packing Bounds: For decoder $\underline{s}$, let $\mathcal{A}_{\underline{s}}$ be the indices of codebooks $C_{A, k}$ decoded at $\underline{s}$. Also let $K_{\underline{s}}$ be the indices $(k, n)$ of codebooks $C_{k, n}$ decoded at $\underline{s}$. Let $\mathcal{A}_{1}$ and $\mathcal{A}_{2}$ partition $\mathcal{A}$, and $K_{1}$ and $K_{2}$ partition $K$. For all such sets, we have the following packing bounds:

$$
\begin{gathered}
H\left(V_{\mathcal{A}_{1}}, U_{K_{1}} \mid V_{\mathcal{A}_{2}}, U_{K_{2}}\right) \leq \sum_{(k, n) \in K_{1}}\left(H\left(U_{k, n}\right)+\rho_{k, n}-r_{k, n}\right) \\
\quad+\sum_{(A, j) \in \mathcal{A}_{1}}\left(H\left(V_{A, j}\right)+\left(\sum_{i \in \underline{s}} \rho_{A, j, i}\right)-r_{A, j}\right) .
\end{gathered}
$$

The resulting $\mathrm{RD}$ vector is:

$$
\begin{aligned}
R_{i} & =\sum_{(A, j) \in \mathcal{S}, i \in A} \rho_{A, j, i}+\sum_{(k, i) \in \mathcal{T}} \rho_{k, i} \\
D_{\underline{s}} & =E\left\{d_{\underline{s}}\left(h_{\underline{s}}\left(U_{\mathcal{A}_{\underline{s}}}, V_{K_{\underline{s}}}\right), X\right)\right\}
\end{aligned}
$$

\section{LineAR CODING EXAMPLES}

In this section, we present two examples showing linear codes achieve points outside of the CMS with binning achievable RD regions.

\section{A. A Three User Example}

Figure 2 depicts the three-descriptions problem. Here $X$ and $Z$ are independent binary-symmetric-sources (BSS). The distortion measure at individual decoders (i.e. decoders 1,

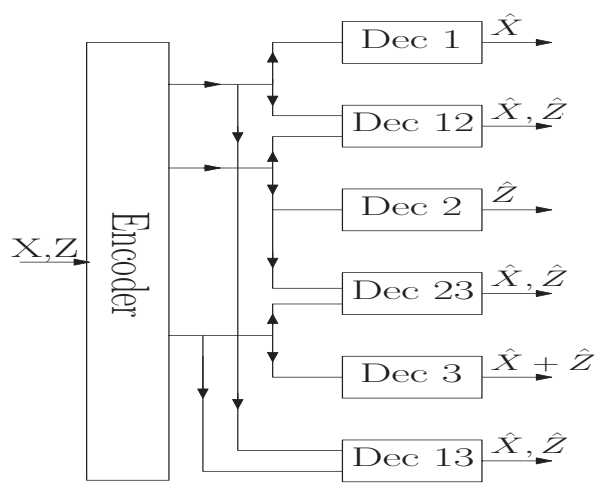

Fig. 2. Three-Descriptions Example

2 and 3) is Hamming distortion. The distortion function at decoders 12,13 , and 23 is

$$
d_{X Z}((\hat{X}, \hat{Z}),(X, Z))=d_{H}(\hat{X}, X)+d_{H}(\hat{Z}, Z) .
$$

Where $d_{H}$ is the Hamming distortion. Let $P_{X Z \hat{X} \hat{Z}}$ be the joint probability distribution of the source, along with two quantizations $\hat{X}=X+N_{\delta}$ and $\hat{Z}=Z+N_{\delta}^{\prime}$, where $N_{\delta}$ and $N^{\prime}{ }_{\delta}$ are $B e(\delta)$ (i.e. with $P\left(N_{\delta}=1\right)=\delta$ ) and independent of all other RV's. $P_{X Z \hat{X} \hat{Z}}$ achieves rate-distortion in a pointto-point (PtP) setting at $D=2 \delta$ and $R=I(\hat{X}, \hat{Z} ; X, Z)=$ $2\left(1-h_{b}(\delta)\right)$.

Theorem 1: In the above MD problem, linear codes can achieve the following rate-distortions:

$$
\begin{aligned}
& R_{i}=1-h_{b}(\delta), D_{1}=D_{2}=\delta, D_{3}=\delta * \delta \\
& D_{12}=D_{13}=D_{23}=2 \delta
\end{aligned}
$$

Proof:

Encoding: Define $r=1-h_{b}(\delta)$. Let $C_{r n \times n}$ be a family of linear codes, which quantize a BSS to Hamming distortion $\delta+\lambda_{n}$, for some $\lambda_{n} \rightarrow 0$. Let $G_{r n \times n}$ be the generator matrices for these linear codes. Define $U_{1}^{n}$ as the quantization of $X^{n}$ using $C_{r n \times n}$ (i.e. $U_{1}^{n}=\operatorname{argmin}_{\hat{x}^{n}}\left\{d_{H}\left(X^{n}, \hat{x}^{n}\right) \mid \hat{x}^{n} \in C_{r n \times n}\right\}$ ). Also define $U_{2}^{n}$ to be the quantization of $Z^{n}$ using the same code. Since the code is linear, $U_{1}^{n}+U_{2}^{n}$ is also a codeword. The first description carries the index of $U_{1}^{n}$, the second description carries the index of $U_{2}^{n}$ and the third description sends the index of $U_{1}^{n}+U_{2}^{n}$.

Decoding: The first and second decoders, receive $U_{1}^{n}$ and $U_{2}^{n}$ respectively, so they satisfy their distortion constraints. Decoder 3 reconstructs $U_{1}^{n}+U_{2}^{n}$. It is straightforward to show that $\frac{1}{n} E\left(d_{H}\left(U_{1}^{n}+U_{2}^{n}, X^{n}+Z^{n}\right) \rightarrow \delta * \delta\right.$. Decoder 12 receives $U_{1}^{n}$ and $U_{2}^{n}$, so it satisfies its distortion requirements. Also decoders 13 and 23 can recover $U_{2}^{n}$ and $U_{1}^{n}$ by adding $U_{1}^{n}+U_{2}^{n}$ to $U_{1}^{n}$ and $U_{2}^{n}$ respectively.

Remark 1: The key to achieving the RD vector, is that due to the linearity of the code, $U_{1}^{n}+U_{2}^{n}$ is in the codebook, so it can be sent on the third description, with the same rate as $U_{i}^{n}$.

Theorem 2: The rate-distortions in theorem 1 are not achievable using the CMS with binning scheme. 


$\begin{array}{llll} & C_{23,2} & & \\ & C_{23,1} & & \\ & C_{13,2} & & \\ & C_{13,1} & C_{3} & C_{2,3} \\ C_{123,2} & C_{12,2} & C_{2} & C_{2,2} \\ C_{123,1} & C_{12,1} & C_{1} & C_{2,1}\end{array}$

Fig. 3. CMS with binning for three descriptions

Proof: We assume that the RD vector is achievable, and arrive at a contradiction. Due to space limitations, a summary of the proof is presented here. The complete proof can be found in [15]. Figure 3 shows the codebooks present in CMS with binning for the three descriptions problem.

Step 1: It is straightforward to check that $\rho_{123,2,1}, \rho_{123,2,2}$, $\rho_{12,2,1}, \rho_{13,2,1}, \rho_{12,2,2}, \rho_{23,2,2}, \rho_{2,1}$ and $\rho_{2,2}$ are 0 . The intuitive reason is that decoder 1 receives the first description at optimal PtP rate-distortion, hence the first description can't carry any indices which are not used in decoder 1 . The same argument holds for decoder 2. This does not mean the codebooks relating to these binning rates are empty. We can only conclude that no bin indices relating to the above codebooks are sent through the corresponding descriptions.

Step 2: The random variables decoded at decoder 1 and decoder 2 are independent of each other. The reason is that decoder 12 is operating optimally; any correlation between descriptions 1 and 2 would be redundant and would contradict optimality. To prove the above statement, a more general situation is investigated in lemma 1 in [15]. The lemma proves that there is no common codebook decoded at decoders 1 and 2, so $C_{123,1}=C_{12,1}=\phi$. Also, using the optimality at decoder 12, it is shown in [15] that the codebooks $C_{12,2}$, $C_{123,2}, C_{2,1}$ are redundant and can be eliminated.

Step 3: Decoder 23 is operating at PtP rate-distortion. $C_{13,2}$ is carried by description 3 through $\rho_{23,2,3}$ but not decoded in decoder 23 , so $\rho_{23,2,3}=0$. Similarly $\rho_{13,2,3}=0$.

Step 4: Since $C_{13,2}$ and $C_{23,2}$ are not sent through any descriptions, one can prove that they are redundant.

Step 5: In this step we show that $\rho_{13,1,1}=\rho_{13,1,3}=\rho_{23,1,2}=$ $\rho_{23,1,3}=0$. Assume $\rho_{13,1,1}>0, V_{13,1}$ is decoded at decoder 3 , so $V_{13,1}$ can be decoded using the third description. As a result $\rho_{13,1,1}$ could be set to 0 without any added distortion at decoder 13. This contradicts optimality at the decoder. Since $C_{13,1}$ and $C_{23,1}$ are not carried by any descriptions, they can be eliminated.

Step 6: The remaining codebooks are $C_{1}, C_{2}, C_{3}$ and $C_{2,3}$. In [15], it is shown that the relation $X, U_{1} \perp Z, U_{3}, U_{2,3}$ must hold. Let $g\left(U_{1}, U_{3}, U_{2,3}\right)$ be the reconstruction of $Z$ at decoder 13 , then the following equation holds.

$$
\begin{aligned}
& \sum_{z, u_{1}, u_{3}, u_{2,3}} p\left(z, u_{1}, u_{3}, u_{2,3}\right) d_{H}\left(g\left(u_{1}, u_{3}, u_{2,3}\right), z\right) \leq \delta \rightarrow \\
& \rightarrow \sum_{u_{1}} p\left(u_{1}\right) \sum_{z, u_{3}, u_{2,3}} p\left(z, u_{3}, u_{2,3}\right) d_{H}\left(g\left(u_{1}, u_{3}, u_{2,3}\right), z\right) \leq \delta
\end{aligned}
$$

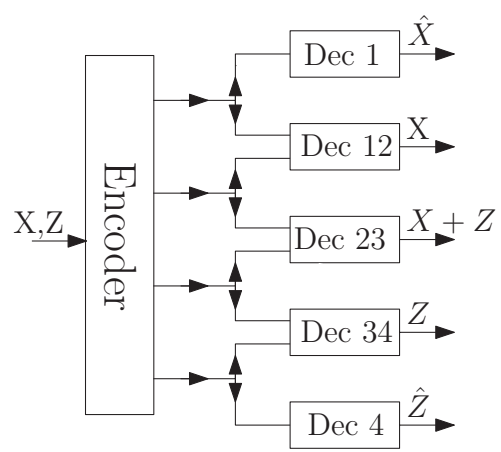

Fig. 4. Four-descriptions example

So there is at least one $u_{1} \in \mathcal{U}_{1}$ such that $\sum_{z, u_{3}, u_{2,3}} p\left(z, u_{3}, u_{2,3}\right) d_{H}\left(g\left(u_{1}, u_{3}, u_{2,3}, z\right) \leq \delta\right.$. Let $g_{u_{1}}\left(U_{3}, U_{2,3}\right)=g\left(u_{1}, U_{3}, U_{2,3}\right)$ be the reconstruction of $\mathbf{Z}$ using $U_{3}$ and $U_{2,3}$. By the same argument we can find a reconstruction of $\mathrm{X}$. Using this it can be proved that decoder 3 can reconstruct both $X$ and $Z$ with Hamming distortion less than $\delta$. This contradicts $R_{3}=1-h_{b}(\delta)$.

\section{B. A Four-Descriptions Example}

So far, it was shown that linear codes outperform the CMS with binning scheme in the three-descriptions problem. The gains present themselves due to the fact that linear codes can compress sums of binary RV's more efficiently. The second example involves a four-descriptions problem. We argue that the gains achieved in this example result from using linear codes which are good source codes as well as good channel codes. The set-up is depicted in figure 4. Here $X$ and $Z$ are BSS's which are related to each other through a $\operatorname{BSC}(\delta)$ (i.e. $X=Z+N_{\delta}$ where $N_{\delta}$ is $B e(\delta)$ and independent of $X$ and $Z$ ). Decoder 1 reconstructs $X$ with Hamming distortion $\delta$, decoder 4 reconstructs $Z$ with the same distortion, the rest of the reconstructions are lossless as shown in the figure.

Theorem 3: For the above distortions, linear codes achieve the following rates:

$$
R_{1}=R_{4}=1-h_{b}(\delta), R_{2}=R_{3}=h_{b}(\delta)
$$

Proof: Assume that $C_{r n \times n}$ is a family of codes which are both good channel codes for a $\operatorname{BSC}(\delta)$, and good source codes for quantizing a BSS to Hamming distortion $\left(\delta+\lambda_{n}\right)$, where $\lambda_{n} \rightarrow 0$. The existence of such codes can be proved using a simple Shannon type argument.

Encoding: The encoder quantizes $X^{n}$ using $C_{r n \times n}$ to $U^{n}$ and sends the index on description 1. It also quantizes $Z^{n}$ using the same code to $V^{n}$ and sends the index on description 4 . The quantization noise at decoder $1, X^{n}+U^{n}$, is sent on description 2, also the quantization noise at decoder 4 is sent on description 3. The required rates are the same as the ones in the theorem.

Decoding: Decoders 1 and 4 receive their desired quantizations. Decoder 12 adds $U^{n}$ to the quantization noise to recover $X^{n}$ losslessly. Decoder 34 recovers $Z^{n}$ in the same manner. Decoder 23 receives the two quantization noises. It then adds 
the two to get $(X+Z)^{n}+(U+V)^{n}$. Since the code is a good channel code for $B S C(\delta)$, the decoder can recover $(U+V)^{n}$ by treating $X+Z$ as channel noise. The decoder can reconstruct $(X+Z)^{n}$ noiselessly by subtracting $(U+V)^{n}$.

Remark 2: Note that with rate exactly $1-h_{b}(\delta)$ the code can only be a good channel code for channels with crossover probability strictly less than $\delta$, while there is a suitable fix to this issue, one can bypass it by assuming that $X+Z$ is $B e(\delta-\lambda)$, where $\lambda$ is a small positive number.

Remark 3: The linearity of the codebook, along with it being a good channel code and a good source code are crucial for achieving this RD vector.

Theorem 4: CMS with binning does not achieve the RD vector in theorem 3 .

Proof: The scheme uses 39 codebooks for the fourdescriptions problem, however, in the special case which we are considering a large number of decoders are not present, so the codebooks can be eliminated in a straightforward fashion Step 1: Any codebook which is not decoded at decoders $1,4,12,23$ and 34 is redundant. For example there are no decoders receiving more than two descriptions, so any codebook which is decoded only when three or more descriptions are available is redundant.

Step 2: By the same kind of argument as in theorem 2, it can be shown that there is no common codebook between decoders 12 and 34. also by the same arguments as in step 2 of the last part, $C_{2,1}$ and $C_{2,4}$ can be eliminated.

Step 3: Since decoders 2 and 3 are not present, $C_{2}$ and $C_{3}$ can be replaced by $C_{2,2}$ and $C_{2,3}$.

Step 4: By the same arguments as in step 3 and 4 of the last proof, $C_{123,2}$ and $C_{234,2}$ are not sent through any description, so they can be eliminated.

Step 5: The 8 remaining codebooks are $C_{1}, C_{2,2}, C_{2,3}, C_{4}$, $C_{12,1}, C_{12,2}, C_{34,1}$ and $C_{34,2}$. In [15] we show that the last four codebooks are not transmitted on any descriptions. It is concluded that $C_{12,2}, C_{34,1}$ and $C_{34,2}$ can be eliminated.

Step 6: It can be proved that the long Markov chain $U_{2,2} \leftrightarrow$ $X \leftrightarrow Z \leftrightarrow U_{2,3}$ must hold between the remaining RV's. The intuitive explanation is that since decoder 12 is operating at optimal RD, $U_{2,2}$ must be independent of all other random variables given $X$. The same argument holds for $U_{2,3}$. We get an inner bound for $R_{2}+R_{3}$ at decoder 23:

$$
R_{2}+R_{3} \geq \min \left(I\left(U_{2,2}, U_{2,3} ; X, Z\right)\right)
$$

Where the minimum is taken over all $P_{U_{2,2}, U_{2,3} \mid X, Z}$ for which the long Markov chain is satisfied and $\left(U_{2,2}, U_{2,3}\right)$ produce a lossless reconstruction of $X+Z$. This resembles the distributed source coding problem in [7]. By the converse in that paper $R_{2}+R_{3}>2 h_{b}(\delta)$. So the RD vector can't be achieved using random codes.

\section{RANDOM CODING IMPROVEMENTS}

In this section we illustrate that CMS with binning can be improved by including additional randomly generated codebooks. For example the scheme does not include a codebook

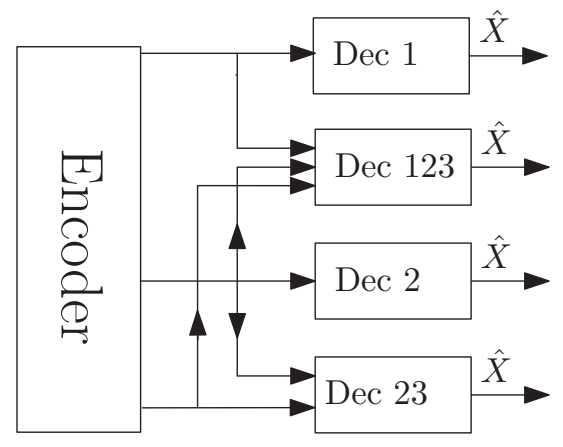

Fig. 5. Three descriptions exmaple for CMS with binning

which is decoded when either description 1 or both descriptions 2 and 3 are received. In the situation depicted in figure 5, the addition of such a codebook results in a larger achievable RD region. Here decoders 1, 23 and 123 have Hamming distortion constraints. The distortion constraint in decoder 2 will be defined later. If decoder 2 is omitted, the example would become equivalent to the two descriptions problem discussed in [1] by combining descriptions 2 and 3 into one description. In that paper, it was proved that the presence of a codebook decoded at all decoders would result in gains in achievable RD. Let $\mathcal{P}=\left\{P_{X_{0}, X_{1}, X_{2}, X}\right\}$ be the set of optimizing distributions in the Zhang-Berger RD region in [1], for a given $R, D$ and $D_{0} . X_{0}$ is the RV relating to the common codebook in that problem. Define $P=\operatorname{argmin}\left(I\left(X ; X_{0}\right)\right)$, where the minimum is taken over all $P_{X_{0}, X_{1}, X_{2}, X} \in \mathcal{P}$. Let $\hat{X}_{0}$ be an RV such that:

$$
P\left(\hat{X}_{0}=\hat{x}_{0} \mid X_{0}=x_{0}\right)= \begin{cases}p & \hat{x}_{0}=x_{0} \\ \frac{1-p}{\left|\mathcal{X}_{0}\right|-1} & \text { O.W. }\end{cases}
$$

Define $P_{\hat{X}_{0}, X}$ based on $P_{\hat{X}_{0}, X_{0}}$ and the Markov chain $\hat{X}_{0} \leftrightarrow$ $X_{0} \leftrightarrow X$. The distortion function at decoder 2 is defined such that $P_{\hat{X}_{0}, X}$ is an optimizing distribution for the distortion function in a PtP setting, such a distortion function can be constructed by the method in [10]. We are interested in achieving the following $\mathrm{RD}$ vector:

$$
\begin{aligned}
& R_{1}=R, R_{2}=I\left(\hat{X}_{0} ; X\right), R_{3}=R-I\left(\hat{X}_{0} ; X\right) \\
& D_{1}=D_{23}=D, D_{2}=d, D_{123}=D_{0} .
\end{aligned}
$$

Where $d=E_{P_{\hat{X}_{0}, X}}\left(d_{12}\left(\hat{X}_{0}, X\right)\right)$.

Theorem 5: The above RD vector is achievable using the CMS with binning scheme with the additional codebook included.

Proof: Define $C^{\prime}$ as the codebook decoded only at decoders 1,23 and 123 . Let the underlying random variable for $C^{\prime}$ be $U^{\prime}$. Define $N_{p}=X_{0}+\hat{X}_{0}$ where the addition is modulo $\left|\mathcal{X}_{0}\right|$. The above $\mathrm{RD}$ vector can be achieved by taking $U_{1}=X_{1}, U_{2}=X_{0}+N_{p}, U^{\prime}=\left(X_{0}, X_{2}\right)$, where the distribution on $X_{1}, X_{2}$ and $X_{0}$ is $P$. 
Theorem 6: The RD vector in (1) is not achievable using CMS with binning.

Proof: Due to space limitations we only provide a summary of the proof. The common codebooks between decoders 1 and 23 are $C_{12,1}, C_{13,1}$ and $C_{123,1}$. Furthermore since decoder 3 is not present, $C_{123,1}$ is the same as $C_{12,1}$ and can be eliminated. We conclude that the common RV must either be sent through $C_{12,1}$ or $C_{13,1}$. Since decoder 2 is operating at optimal PtP rate-distortion, descriptions 2 and 3 can't time-share in transmitting $X_{0}$ on $C_{12,1}$ and $C_{13,1}$. Let $R_{0}$ be the rate of the common component then by construction $R_{0} \geq I\left(X, X_{0}\right)$. So $X_{0}$ can't be transmitted on either of $C_{12,1}$ and $C_{13,1}$, which is a contradiction.

\section{Linear Coding Achievable Region}

In this section we provide an inner bound to the achievable $\mathrm{RD}$ region using linear codes.

Theorem 7: RD vectors satisfying the following bounds are achievable using linear codes.

$$
H\left(V_{\mathcal{A}}, U_{K} \mid X\right) \geq \sum_{(A, j) \in \mathcal{A}}\left(q-\rho^{\prime}{ }_{A, j}-r_{A, j}\right)+\sum_{(k, n) \in K}\left(q-\rho_{k, n}^{\prime}-r_{k, n}\right)
$$$$
H\left(V_{\mathcal{A}_{1}}, U_{K_{1}} \mid V_{\mathcal{A}_{2}}, U_{K_{2}}\right) \leq \sum_{(k, n) \in K_{1}}\left(q-\rho_{k, n}^{\prime}+\rho_{k, n}-r_{k, n}\right)
$$$$
\left.+\sum_{(A, j) \in \mathcal{A}_{1}}\left(q-\rho^{\prime}{ }_{A, j}+\left(\sum_{i \in \underline{s}} \rho_{A, j, i}\right)-r_{A, j}\right)\right)
$$

$\rho_{A, j}^{\prime} \leq q-H\left(V_{A, j}\right)$

$\rho_{k, n}^{\prime} \leq q-H_{U_{k, n}}$.

Here $q$ is the maximum of the cardinality of all RV's involved in the optimization. Also $\underline{s}, \mathcal{A}_{\underline{s}}, K_{\underline{s}}, \mathcal{A}_{1}, \mathcal{A}_{2}, K_{1}$ and $K_{2}$ are defined in previous sections.

Furthermore if the encoder wants to transmit the sum of two random variables $Y, Z \in\left\{U_{A, j}, V_{k, n}\right\}$, the following covering bound must hold:

$$
\max \left\{r_{y}, r_{z}\right\} \geq q-H(Y+Z)
$$

The packing bounds are also affected. If decoder $\underline{s}$ receives the bin index of $Y+Z$, then we have three cases:

Case 1: Decoder $\underline{s}$ reconstructs both $Y$ and $Z$. In this case, in the packing bound corresponding to this decoder, $\rho_{Y}$ is replaced with $\rho_{Y}+t \rho_{Y+Z}$ and $\rho_{Z}$ is replaced with $\rho_{Z}+(1-$ t) $\rho_{Y+Z}$, where $t \in[0,1]$ and $\rho_{Y+Z}$ is the rate with which the codebook for $Y+Z$ is binned.

Case 2: The decoder only reconstructs $\mathrm{Y}$ (or Z), in which case reconstructing $Y+Z$ is the same as reconstructing $(Y, Z)$. The packing bounds are written as if $Z$ was sent to the decoder with binning rate $\rho_{Y+Z}$.

Case 3: The decoder does not reconstruct $Y$ or $Z$. In this case in the packing bound $U_{\mathcal{A}}$ is replaced with $\left(Y+Z, U_{\mathcal{A}}\right)$.

Remark 4: If $Y+Z$ is taken to be trivial, the above bound reduces to the CMS with binning achievable region.
Remark 5: $q, \rho_{A, j}^{\prime}$ and $\rho^{\prime}{ }_{k, n}$ are eliminated after the Fourier-Motzkin elimination and do not play a role in determining the achievable region.

Remark 6: The above rate region can be improved upon by adding the extra codebooks mentioned in the last section, and also by allowing reconstruction of multi-variate summations of the random variables.

\section{CONCLUSiON}

A new coding scheme for the general MD problem was proposed. It was shown that the scheme outperforms previous known random coding strategies. An example was given illustrating that the CMS with binning scheme can also be improved by including additional randomly generated codebooks.

\section{REFERENCES}

[1] Z. Zhang, T. Berger, "New results in binary multiple-descriptions," IEEE Transactions on Information Theory, vol.33, no.4, pp.502,521, Jul 1987

[2] R. Venkataramani, G. Kramer, and V.K. Goyal, "Multiple description coding with many channels," IEEE Transactions on Information Theory, vol. 49, no. 9, pp. 21062114, 2003.

[3] K. Viswanatha, E. Akyol, and K. Rose, "Combinatorial message sharing for a refined multiple-descriptions achievable region," in IEEE International Symp. on Information Theory. IEEE, pp. 13121316., 2011.

[4] S.S. Pradhan, R. Puri, and K. Ramchandran, "n-channel symmetric multiple descriptions-part i:(n, k) source-channel erasure codes," IEEE Transactions on Information Theory, vol. 50, no. 1, pp. 4761, 2004.

[5] Tian, C.; Jun Chen, "New Coding Schemes for the Symmetric K Description Problem," IEEE Transactions on Information Theory, vol.56, no. 10 , pp. 5344,5365 , Oct. 2010

[6] E. Akyol, K. Viswanatha, K. Rose, "Combinatorial message sharing and random binning for multiple description coding," in IEEE International Symp. on Information Theory. IEEE, pp.1371,1375, 1-6 July 2012.

[7] J. Korner and K. Marton, "How to encode the modulo-two sum of binary sources," IEEE Transactions on Information Theory, vol. 25, no. 2, pp. 219221, 1979.

[8] A. Jafarian, S. Vishwanath, "Gaussian Interference Networks: Lattice Alignment", IEEE Inf. Th. Workshop, January 2010

[9] A. Padakandla, S.S. Pradhan, "Achievable rate region for three user discrete broadcast channel based on coset codes," in IEEE International Symp. on Information Theory. IEEE, pp.1277,1281, 7-12 July 2013

[10] S.S. Pradhan, J. Chou, K. Ramchandran, "Duality between source coding and channel coding and its extension to the side information case," Information Theory, IEEE Transactions on, vol.49, no.5, pp.1181,1203, May 2003

[11] A.B. Wagner, B.G. Kelly and Y. Altuğ, "Distributed Rate-Distortion With Common Components," IEEE Transactions on Information Theory, vol.57, no.7, pp.4035-4057, July 2011

[12] B. Nazer, M. Gastpar, "Computation over Gaussian Multiple-Access Channels," in IEEE International Symp. on Information Theory. IEEE, pp.2391,2395, 24-29 June 2007

[13] T. Philosof, R. Zamir, "On the Loss of Single-Letter Characterization: The Dirty Multiple Access Channel," IEEE Transactions on Information Theory, vol.55, no.6, pp.2442,2454, June 2009

[14] D. Krithivasan, S.S. Pradhan, "Distributed Source Coding Using Abelian Group Codes: A New Achievable Rate-Distortion Region,” IEEE Transactions on Information Theory, vol.57, no.3, pp.1495,1519, March 2011

[15] F. Shirani, S.S. Pradhan, "An Achievable Rate-Distortion Region for the Multiple Descriptions Problem", available at arxiv.org 